\title{
Lumped Element Model of Single CMUT in Collapsed Mode
}

\author{
Elif Aydogdu*†, Alper Ozgurluk ${ }^{\ddagger}$, H. Kagan Oguz*, Abdullah Atalar*, and Hayrettin Koymen* \\ ${ }^{*}$ Bilkent University, Electrical and Electronics Engineering Department, TURKEY \\ $\ddagger$ University of California Berkeley, Electrical Engineering and Computer Sciences Department, TURKEY \\ †e-mail: aelif@ee.bilkent.edu.tr
}

\begin{abstract}
In this study, an equivalent electrical circuit model for a single circular CMUT in both uncollapsed and collapsed modes is obtained. In order to model the collapsed mode mechanics, the governing differential equation is solved semi-analytically for a large number of normalized cases. Then the calculations are adapted to the equivalent electrical circuit model. The model uses the self radiation impedance of both uncollapsed and collapsed modes. The model is fully parametric in such a way that a CMUT cell of given dimensions and parameters can be simulated under an arbitrary large signal excitation. Transient simulations can be performed in less than a minute with a circuit simulator. Transient and frequency domain simulations are consistent with finite element analysis results.
\end{abstract}

\section{INTRODUCTION}

Capacitive micromachined ultrasonic transducer (CMUT) is shown to be an efficient transmitter in collapsed mode [1], [2]. However, this mode is not commonly used as its operation is highly nonlinear and cannot be predicted through simple analytical calculations or lumped element simulations. In [2] it is observed that collapsed mode operation is very sensitive to any change in physical dimensions. In order to work in this mode, we need a fast and parametric simulation tool that helps us to understand the effects of all parameters and optimize the operation.

Especially, the array simulations have very high computation cost for finite element analysis (FEA), and large arrays cannot be simulated in FEA environment. For the uncollapsed mode, lumped element modeling is a fast and accurate alternative to FEA [3]. However, there is no parametric lumped element model for collapsed mode as its lumped parameters cannot be defined analytically.

Previously an equivalent circuit model for a CMUT in collapsed mode was given in [4], but it was specific to a single CMUT design, and it contained inaccuracies because of the uniform force approximation in deflection formulation.

In this work, we obtain a parametric equivalent circuit model of a single CMUT cell for both uncollapsed and collapsed modes. We solve the differential equation [5] governing the static deflection of a collapsed membrane without using the uniform force approximation. Since the electrical force and deflection depend on each other, the differential equation is solved iteratively until the solution converges. We adapt the results of calculations to the lumped element model using polynomial fitting. The self radiation impedance of uncollapsed and collapsed modes [6] is also included in the model.

\section{Equivalent Electrical Circuit Model of CMUT}

Our equivalent electrical circuit model for CMUT using rms through and across variables is given in Fig.1. The

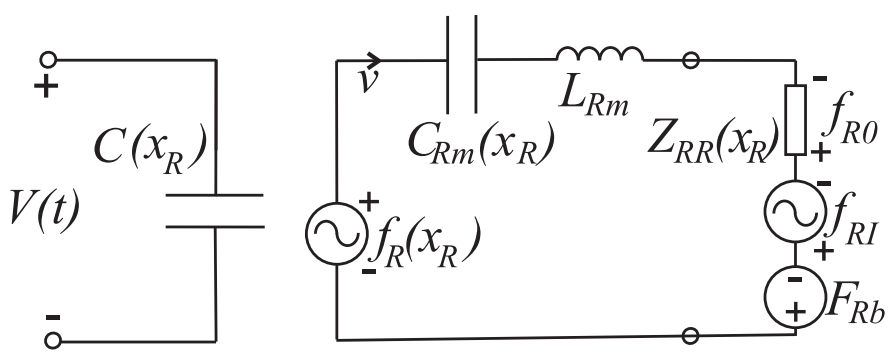

Fig. 1. The equivalent electrical circuit model of CMUT.

electrical port is on the left hand side, where the CMUT appears as a nonlinear capacitor. The CMUT capacitance $(C)$ depends instantaneously on the membrane displacement $x_{R}{ }^{1}$. On the mechanical (right) side, the $r m s$ velocity, $v_{R}$, is the through variable and the force, $f_{R}$, is the across variable. $C_{R m}$ is the membrane compliance, $L_{R m}$ is the membrane mass, $f_{R 0}$ is the force on the medium impedance, $f_{R I}$ is the force of incoming acoustic signals, and $F_{R b}$ is the force due to static medium pressure, all values suitable for $r m s$ quantities.

For the uncollapsed mode, simple approximations let us express $C$ and $f_{R}$ as analytical functions of $x_{R}$ [7], and the compliance has a fixed value in this mode:

$$
C_{R m_{0}}=\frac{9}{80} \frac{\left(1-v^{2}\right) a^{2}}{\pi E t_{m}^{2}}
$$

In the collapsed mode, analytical expressions cannot be obtained for capacitance and electrical force. The compliance is no longer constant and it also cannot be expressed analytically. In order to obtain the collapsed mode model, we solve for a number of normalized cases of static excitation as described in the following section, and use the results to define the lumped elements. 


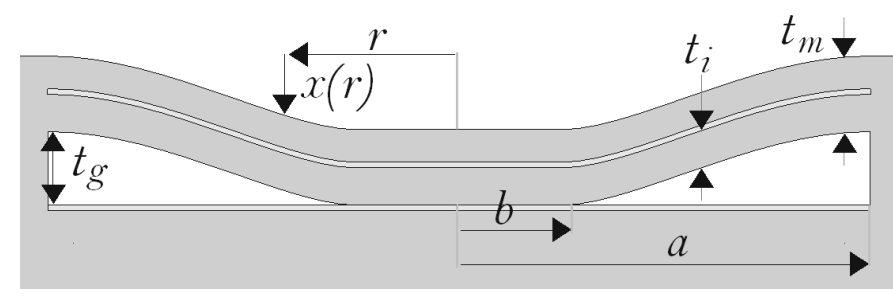

Fig. 2. The dimensional parameters shown on collapsed CMUT membrane

\section{A. Solution of the Differential Equation for the Deflection Profile}

The deflection profile $x(r)$ of the collapsed mode circular CMUT membrane shown in Fig.2 is formulated in [5]:

$r \frac{d}{d r}\left(\frac{1}{r} \frac{d}{d r}\left(r \frac{d x(r)}{d r}\right)\right)=\frac{1}{D} \int_{b}^{r}\left(P_{b}+\frac{\epsilon_{0} V^{2}}{2\left(t_{g}-x(r)\right)^{2}}\right) r d r$

The relevant parameters are given in Table I.

TABLE I. CMUT PARAMETERS

\begin{tabular}{|c|l|}
\hline$P_{b}$ & uniformly distributed pressure \\
\hline$V$ & input voltage \\
\hline$\epsilon_{0}$ & dielectric permittivity of air \\
\hline$\epsilon_{r}$ & relative dielectric permittivity of insulator \\
\hline$t_{g e}$ & $=t_{g}+t_{i} / \epsilon_{r}$, effective gap height \\
\hline$E$ & Young's modulus of membrane material \\
\hline$v$ & Poisson's ratio of membrane material \\
\hline$\rho$ & density of membrane material \\
\hline$D$ & $=E t_{m}^{3} / 12\left(1-v^{2}\right)$, flexural rigidity of membrane material \\
\hline
\end{tabular}

In uncollapsed mode, to obtain an analytical solution for $x(r)$ in (2), the electrical force distribution can be approximated with a uniform pressure. In collapsed mode, the uniform pressure approximation brings inaccuracy [4], as the electrical force is highly nonuniform. So we utilize the electrical force distribution in our calculations, and solve (2) numerically with an iterative algorithm. We calculate deflection profiles of many possible normalized CMUT designs, for various voltage and pressure values, and use those calculations to come up with a dataset defining the lumped element characteristics.

In order to normalize the problem, we rearrange (2) to

$$
\bar{r} \frac{d}{d \bar{r}}\left(\frac{1}{\bar{r}} \frac{d}{d \bar{r}}\left(\bar{r} \frac{d}{d \bar{r}} \bar{x}(\bar{r})\right)\right)=\int_{\bar{b}}^{\bar{r}} 64\left(\frac{P_{b}}{P_{g}}+\frac{2\left(V / V_{r}\right)^{2}}{9(1-\bar{x}(\xi))^{2}}\right) \xi d \xi
$$

where

$$
\bar{r}=\frac{r}{a}, \quad \bar{b}=\frac{b}{a}, \quad \bar{x}(.)=\frac{x(.)}{t_{g e}} .
$$

In this differential equation, there are only three variables; normalized input voltage $V / V_{r}$, normalized static pressure $P_{b} / P_{g}$, and normalized gap height $t_{g} / t_{g e}$ that determine the profile. $t_{g e}=t_{g}+t_{i} / \epsilon_{r}$ is the effective gap height, $V_{r}$ is the collapse voltage at vacuum, and $P_{g}$ is the static pressure required for a peak deflection of $t_{g e}$ with zero voltage input:

$$
P_{g}=\frac{64 D t_{g e}}{a^{4}}, \quad V_{r}=\frac{16}{3 a^{2}} \sqrt{\frac{D t_{g e}^{3}}{\epsilon_{0}}} .
$$

\footnotetext{
${ }^{1} x_{R}=1 /\left(\pi a^{2}\right) \sqrt{\int_{0}^{a} 2 \pi r x^{2}(r) d r}$ is the root-mean-square (rms) displacement as a lumped variable of radius dependent displacement $x(r)$
}

The deflection profile of any CMUT design can be calculated using the results obtained with normalized formulation, by transforming the parameters to the normalized basis through simple arithmetic operations.

\section{B. Extended CMUT Biasing Chart}

The calculations carried out for $P_{b}=0$ are given on Fig.3. The normalized $r m s$ displacement $\bar{x}_{R}=x_{R} / t_{g e}$ is plotted as a function of normalized input voltage $\left(V_{D C} / V_{r}\right)$. This plot is the CMUT biasing chart of [7] with collapsed mode extension. The curve shown with the dashed line is obtained with the uniform-force approximation. The solid curve is obtained using the electrical force distribution in (3). Here we can see the

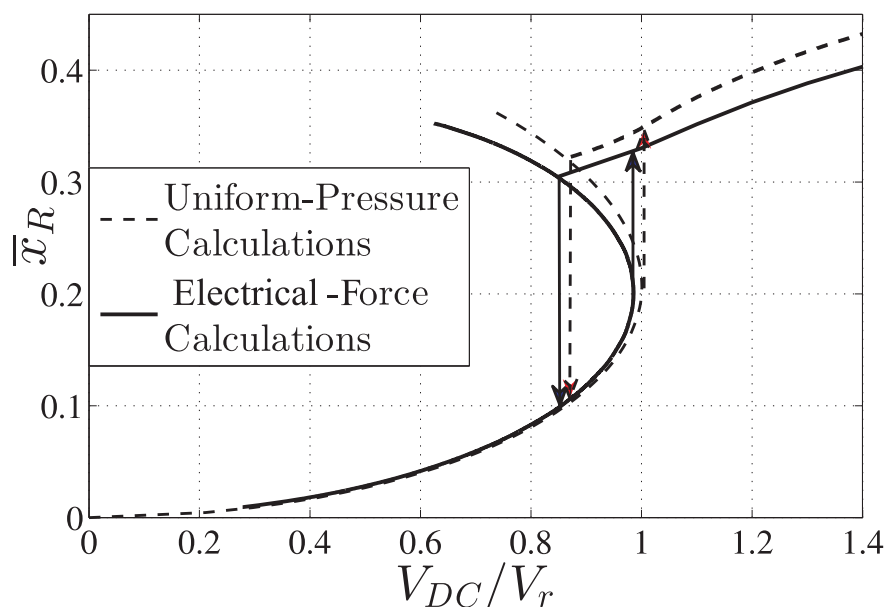

Fig. 3. The extended CMUT biasing chart, normalized static displacement as a function of normalized bias voltage, obtained with uniform pressure calculations and electrical force calculations.

error originating from uniform-force approximation, especially beyond the collapse point.

The profiles of the membranes deflected with only uniform pressure (zero input voltage), and with only electrical force (zero uniform pressure) are given on Fig.4. The upper profile corresponds to the threshold of collapse in the uncollapsed mode. The uniform pressure deflection is very close to electrical force deflection. However we observe significant difference between the two at the snapback (middle), and in collapsed mode (lower). Considering this difference, the

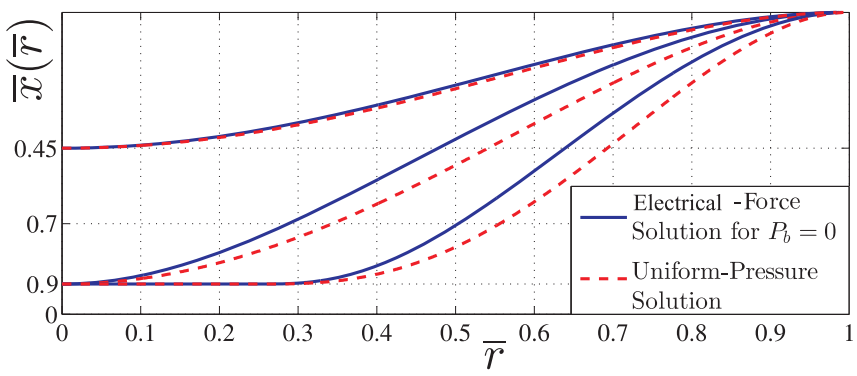

Fig. 4. The deflection profiles obtained with only uniform pressure (dashed) and only electrical force (solid), in uncollapsed mode (upper), at snapback (middle), and in collapsed mode (lower).

uniform-pressure assumption should not be used for electrically collapsed membranes, otherwise simulation accuracy will 
suffer. We use electrical force together with uniform pressure in our calculations to have a high accuracy.

\section{Lumped Elements as a Function of Displacement}

We have analyzed 1400 different cases for $t_{g} / t_{g e}=0.4$, $0.5,0.6,0.65,0.73,0.82$, and 0.9 ; and $P_{b} / P_{g}=0,0.2,1$, and 2 . We used 50 values of $\left(V / V_{r}\right)$ between the normalized snapback voltage and $\left(V / V_{r}\right)=10$. Having found the deflection profile $\bar{x}(\bar{r})$ and $\bar{b}$ for each case, we calculated corresponding $\bar{x}_{R}, C / C_{0}, d C / d \bar{x}_{R}$, and $C_{R m} / C_{R m_{0}}$.

For the normalized profile $\bar{x}(\bar{r})$, the normalized capacitance is calculated with the formula

$$
\frac{C}{C_{0}}=\int_{0}^{1} \frac{2 \bar{r}}{1-\bar{x}(\bar{r})} d \bar{r}
$$

where $C_{0}$ is the undeflected membrane capacitance:

$$
C_{0}=\epsilon_{0} \pi a^{2} / t_{g e} .
$$

We obtained curves of normalized capacitance as a function of $\bar{x}_{R}$, for all values of $t_{g} / t_{g e}$ and $P_{b} / P_{g}$ in collapsed mode. For the uncollapsed mode, we used the capacitance formulation obtained with the uniform-force approximation.

The electrical force of the lumped element model is defined as the energy conserving force of the electrical capacitor:

$$
f_{R}=\frac{d E_{e l}}{d x_{R}}=\frac{V^{2}}{2} \frac{d C}{d x_{R}}=\frac{V^{2}}{2 t_{g e}} \frac{d C}{d \bar{x}_{R}} .
$$

Using the capacitance data, we calculated $d C / d \bar{x}_{R}$ numerically for every value of $\bar{x}_{R}$.

The restoring force of the membrane is determined by its compliance. The compliance of the membrane for each static analysis is calculated through the formula:

$$
C_{R m}=\frac{x_{R}}{F_{R}+F_{R b}} .
$$

The normalized compliance plot is given in Fig. 5. In the stable region of uncollapsed mode it has a fixed value of $C_{R m 0}$. As the membrane gets into unstable region, there is an increase in the compliance. In the collapsed mode the compliance decreases with increasing displacement, as the contact radius increases and the membrane gets stiffer.

The contact radius $b$, is another important parameter as it affects the self radiation impedance. Results of [6] show that the radiation impedance of collapsed CMUT change with contact radius. The normalized contact radius $\bar{b}$, is recorded as a function of $\bar{x}_{R}$, for all values of $t_{g} / t_{g e}$ and $P_{b} / P_{g}$ to be used in radiation impedance calculations.

Polynomials are fitted to all the curves of lumped parameter calculations in order to express their dependence on $\bar{x}_{R}$. The lumped element model is realized in a circuit simulator (ADS from Agilent Tech.) using controlled sources for introducing the polynomials. The self radiation impedance of [6] is also introduced with lookup tables at 13 different values of $\bar{b}$ up to 0.6 .

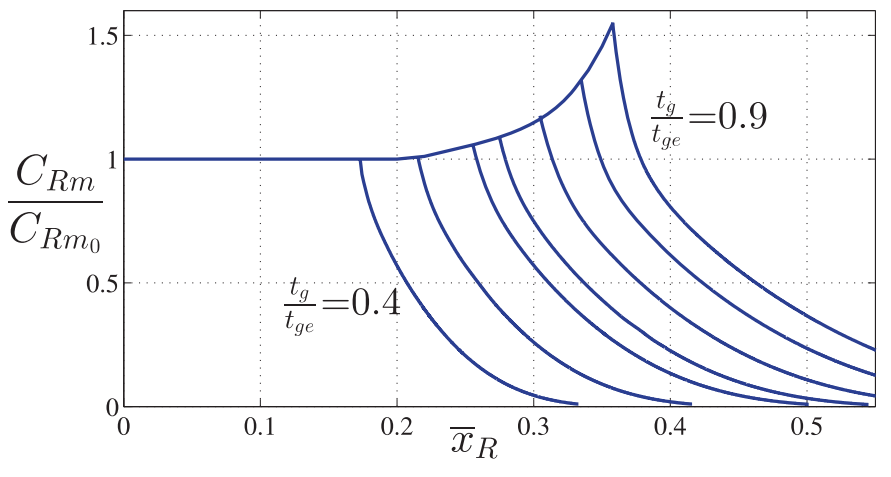

Fig. 5. The membrane compliance normalized to uncollapsed mode value, as a function of normalized $\mathrm{rms}$ displacement. The results for $t_{g} / t_{g e}=0.4$, $0.5,0.6,0.65,0.73,0.82$, and 0.9 are plotted for $P_{b} / P_{g}=0$.

\section{Simulation Results}

The transient analysis of a CMUT cell with parameters given in Table II is shown in Fig. 6. A $0.3 \mu$ s pulse of $50 \mathrm{~V}$ $\left(t_{\text {rise }}=t_{\text {fall }}=10 \mathrm{nsec}\right)$ is applied to the cell. The immersion medium is water and the pressure is $1 \mathrm{~atm} . \bar{x}_{R}$ is plotted as a function of time. As the collapse voltage is smaller than the pulse amplitude, the membrane experiences collapse and snapback. $\bar{x}_{R}=0.51$ corresponds to a collapsed membrane with a collapse radius of $\bar{b}=0.43$. The sudden pull and release of the membrane cause oscillations in the displacement. The collapse oscillations are at a high frequency and they die quickly, since the stiff membrane oscillates at a higher frequency and it experiences a relatively high radiation resistance. On the other hand, the snapback oscillations occur at a lower frequency and the damping is smaller, because after snapback the membrane is no longer stiff and at lower frequencies the radiation resistance is smaller. In the same figure, FEA simulations of the same cell is given displaying a very good agreement.

TABLE II.

\begin{tabular}{|l|l|l|l|}
\hline$E$ & $v$ & $\epsilon_{r}$ & $\rho$ \\
\hline $110 \mathrm{GPa}$ & 0.27 & 5.4 & $3.1 \mathrm{~g} / \mathrm{cm}^{3}$ \\
\hline \hline$a$ & $t_{m}$ & $t_{i}$ & $t_{g}$ \\
\hline $15 \mu \mathrm{m}$ & $0.6 \mu \mathrm{m}$ & $0.2 \mu \mathrm{m}$ & $0.1 \mu \mathrm{m}$ \\
\hline
\end{tabular}

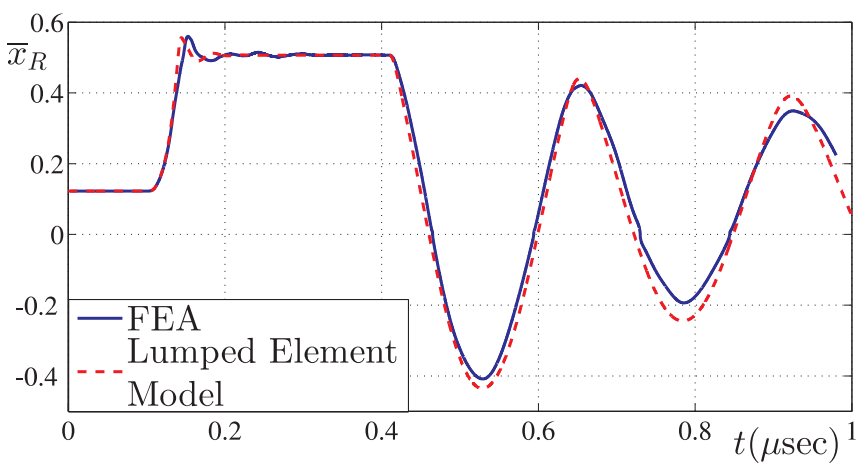

Fig. 6. The normalized rms displacement of a CMUT cell of Table II excited with a $0.3 \mu \mathrm{s}$ long $50 \mathrm{~V}$ pulse of $10 \mathrm{~ns}$ rise and fall times. Lumped element model simulation (dashed) is compared to the FEA result (solid). 
Fig. 7 depicts an analysis of the CMUT cell given in Table III at different levels of bias voltage with a small $\mathrm{AC}$ excitation level of $1 \mathrm{~V}$ peak. The mechanical resonance

TABLE III.

\begin{tabular}{|l|l|l|l|}
\hline$a$ & $t_{m}$ & $t_{i}$ & $t_{g}$ \\
\hline $30 \mu \mathrm{m}$ & $3.2 \mu \mathrm{m}$ & $0.2 \mu \mathrm{m}$ & $0.2 \mu \mathrm{m}$ \\
\hline
\end{tabular}

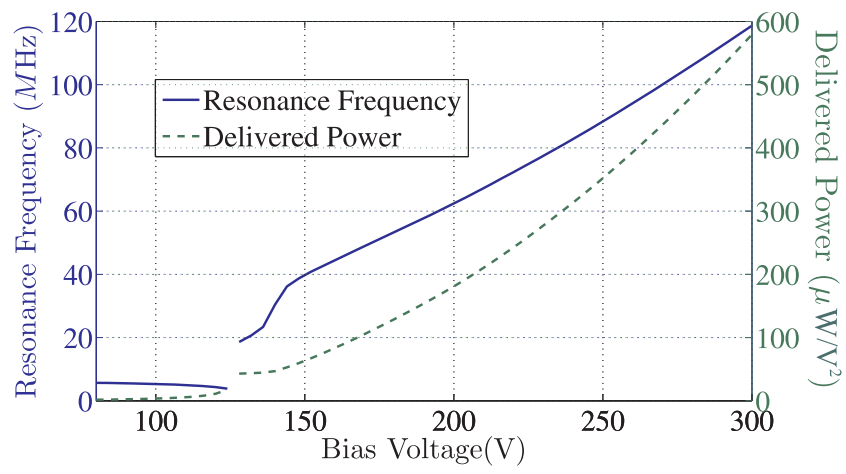

Fig. 7. The mechanical resonance frequency as a function of bias voltage (solid) for the CMUT cell of Table III. The real power delivered to the medium using an $\mathrm{AC}$ excitation voltage of $1 \mathrm{~V}$ peak at the resonance frequency of the cell as a function of the bias voltage (dashed).

frequency of the CMUT cell is plotted at each DC bias value. The resonance frequency is nearly $5 \mathrm{MHz}$ for small bias voltages and it drops slightly as the bias voltage is increased. As soon as the collapse occurs at a bias voltage of $128 \mathrm{~V}$ the resonance frequency jumps to $19 \mathrm{MHz}$. From this point on, the resonance frequency increases almost linearly with the bias voltage as the membrane goes into deep collapse ${ }^{2}$. This result is consistent with experimental observations. [1]

In the same figure, the power delivered to the liquid medium with a small (1V peak) sinusoidal excitation voltage at the corresponding resonance frequency is also plotted as a function of bias voltage. We observe that the power delivered is considerably more in the collapse mode as compared to uncollapsed mode even though the AC excitation amplitude is the same. Moreover, the delivered power gets even bigger as the membrane experiences a deeper collapse. This dependence is also consistent with experimental observations. We note that it is very time consuming to get Fig. 7 using FEA.

\section{CONCLUSION}

A fast and accurate simulation tool for both uncollapsed and collapsed mode operation of CMUT is given. In order to have high accuracy, the collapsed membrane characterization is based on deflection calculations performed utilizing a nonuniform electrical force distribution rather than a uniform pressure. The solution of the normalized differential equation is carried out 1400 times to scan the various parameters of a CMUT membrane. The results are used to determine lumped parameter expressions as polynomials. The proposed model

\footnotetext{
${ }^{2}$ There is a small undulation in the resonance frequency curve around the bias voltage of $140 \mathrm{~V}$. It is because of a variation in the radiation reactance around this point. For frequencies larger than $60 \mathrm{MHz}$, the radiation reactance stays nearly constant and the resonance frequency curve is almost linear.
}

can be realized using a time-domain circuit simulator capable of handling frequency response data.

Simulation results are in good agreement with the corresponding FEA simulations. The transient simulation given in this paper takes less than a minute using the electrical model, decreasing the simulation time more than a hundred times compared to FEA.

\section{REFERENCES}

[1] S. Olcum, Y. Yamaner, A. Bozkurt, H. Koymen, and A. Atalar, "Deep collapse operation of capacitive micromachined ultrasonic transducers," IEEE Trans. Ultrason., Ferroelectr., Freq. Control, vol. 58, pp. 2475$2483,2011$.

[2] K. K. Park, O. Oralkan, and B. T. Khuri-Yakub, "A comparison between conventional and collapse-mode capacitive micromachined ultrasonic transducers in 10-MHz 1-D arrays," IEEE Trans. Ultrason., Ferroelectr., Freq. Control, vol. 60, pp. 1245-1255, 2013.

[3] H. K. Oguz, A. Atalar, and H. Koymen, "Equivalent circuit-based analysis of CMUT cell dynamics in arrays," IEEE Trans. Ultrason., Ferroelectr., Freq. Control, vol. 60, pp. 1016-1023, 2013.

[4] S. Olcum, Y. Yamaner, A. Bozkurt, H. Koymen, and A. Atalar, "An equivalent circuit model for transmitting capacitive micromachined ultrasonic transducers in collapse mode," IEEE Trans. Ultrason., Ferroelectr., Freq. Control, vol. 58, pp. 1468-1476, 2011.

[5] S. Timoshenko and S. W. Woinowsky-Krieger, Theory of Plates And Shells. NY: McGraw Hill, 1959.

[6] A. Ozgurluk, A. Atalar, H. Koymen, and S. Olcum, "Radiation impedance of collapsed capacitive micromachined ultrasonic transducers," IEEE Trans. Ultrason., Ferroelectr., Freq. Control, vol. 59, pp. 1301-1308, 2012

[7] H. Koymen, A. Atalar, E. Aydogdu, C. Kocabas, H. K. Oguz, S. Olcum, A. Ozgurluk, and A. Unlugedik, "An improved lumped element nonlinear circuit model for a circular CMUT cell," IEEE Trans. Ultrason., Ferroelectr., Freq. Control, vol. 59, pp. 1791-1799, 2012. 